\title{
Physiological Changes of Intra Ocular Pressure during First and Third Trimester of Pregnancy
}

\author{
Dr. Pitta Paramjyothi ${ }^{1}$, Dr. A.Krishnaveni ${ }^{2}$ \\ ${ }^{I}$ (Associate Professor of Physiology, Department Of Physiology, Guntur Medical College, Guntur, Dr. NTR \\ University Of Health Sciences, Vijayawada, Andhra Pradesh, INDIA 522004) \\ ${ }^{2}$ (Associate Professor of Physiology, Department Of Physiology, Mamata Medical College, Khammam, Dr. NTR \\ University Of Health Sciences, Vijayawada, Andhra Pradesh, INDIA 507001)
}

Abstract: Pregnancy brings in an increase in hormones that may cause changes in vision by altering the strength of refraction. The temporary changes in vision will return to normal after delivery. The primary objective is to identify the ocular changes in both eyes during first and third trimesters of normal pregnancy by doing ocular examination along with regular antenatal check-ups to detect the blood pressure change, to diagnose pre-eclampsia and other ocular disorders at an early stage. Intra Ocular Pressure of both eyes was measured by Schiotz tonometry.

Keywords: First trimester, Intra Ocular Pressure, Pregnancy, Schiotz tonometry, third trimester

\section{Introduction}

Pregnancy implies progressive anatomical and physiological changes that are not only confined to the reproductive organs but also to all the systems of the body. The tendency of fluid retention affects refraction. As a result, current spectacle (or) contact lenses may temporarily be either too weak or too strong depending upon the specific refractive error.

As the pregnancy affects pre-existing ocular conditions such as diabetic retinopathy, toxemia of pregnancy, tumors and immunological disorders [1] and it can have beneficial effects on one such pre-existing condition as glaucoma.

Very few reports are available to indicate the effect of pregnancy changes on IOP.

Compared with the normo-tensive women, pre-eclampsia women have increased IOP in the peripartum period [2]. Ocular and systemic parasympathetic involvement appears earlier than sympathetic involvement in diabetic patients [3].

Very high systemic BP causes development of hypertensive retinopathy with bleeding in the retina, retinal detachment which may progress to permanent visual impairment [4] if untreated.

Pregnancy involves number of endocrine interactions, so in the present study we tried to observe the physiological changes of IOP in first and third trimesters of pregnancy to be aware of ocular changes to know what symptoms indicate a serious problem.

\section{Materials and Methods}

The work was carried out at Kakatiya Medical College, Warangal, ChandraKantaiah Memorial Hospital, Warangal, Government Maternity Hospital, Hanamkonda and Regional Eye Hospital, Warangal for the selection of subjects. Prior to the commencement of the study approvals of Institute Research Council, Ethical Committees were obtained and each subject was informed in detail of its objective, the aim of the research protocol and the method to be used. Along with routine lab investigations, ante-natal check-ups, local examination of both the eyes was performed.

A total of 30 normotensive pregnant women of age-group 20-30 years (mean 25) during first and third trimester with known Last Menstrual Period, regular ante-natal check-ups and with no history of previous abortion, without any refractive error or systemic disease presently and previously were selected for this study. The changes in IOP were compared between first and third trimesters of pregnancy.

The foot plate of lower end of plunger of Schiotz tonometer (Speedway Surgical Co., Pindi street, Luthiana) was sterilized. After anaesthetizing the cornea with $4 \%$ topical xylocaine, the subject was made to lie supine on a coach and instructed to fix at a target on the ceiling. Then by separating the eye lids with left hand gently, the foot plate of the tonometer is rested vertically on the centre of the cornea. The reading on scale was recorded as soon as the needle becomes steady. The tonometer was lifted and a drop of antibiotic was instilled. A conversion table was then used to derive the IOP in $\mathrm{mm}$ of $\mathrm{Hg}$ from the scale reading and the plunger weight.

Records from both the eyes were analyzed and a mean of them was taken into consideration. The data obtained from pregnant women during first and third trimesters was compared by using unpaired student's t-test for difference of means with unequal variances for statistical analysis. 


\section{Results}

A total of 30 pregnant women during first trimester and third trimester were examined. IOP was significantly affected during first trimester when compared to third trimester. Mean and Standard Deviation of right and left eye IOP during first and third trimesters of pregnancy is being compared in the table.

In our present study there is a decrease in IOP during third trimester of pregnancy when compared to first trimester of pregnancy. The data obtained was analyzed by using unpaired student's t-test. There is a significant gradual decrease in IOP from first trimester to third trimester with $\mathrm{p}$-value $<0.0001$.

Comparison of IOP during First and Third Trimesters of Pregnancy

\begin{tabular}{|l|l|l|l|}
\hline IOP $(\mathrm{mm}$ of $\mathrm{Hg})$ & RIGHT EYE $($ Mean \pm SD) & LEFT EYE $($ Mean \pm SD) & p-Value \\
\hline FIRST TRIMESTER & $14 \pm 0.68$ & $13.56 \pm 0.87$ & $<0.0001$ \\
\hline THIRD TRIMESTER & $11.65 \pm 0.91$ & $11.24 \pm 0.89$ & $<0.0001$ \\
\hline
\end{tabular}

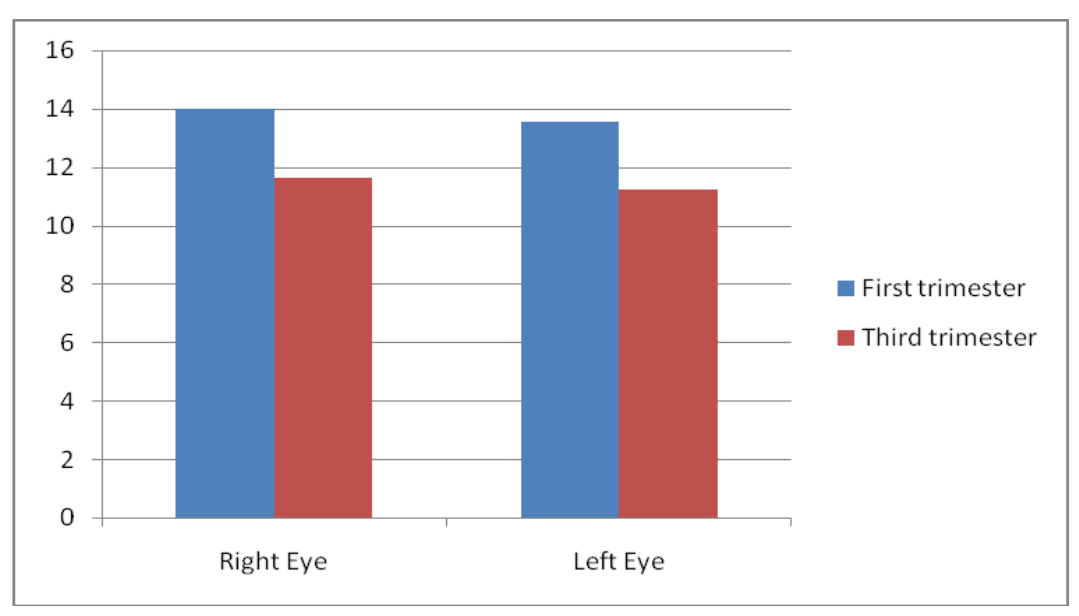

\section{Conclusion}

The decrease in IOP during first trimester to third trimester of pregnancy is due to increased facility of aqueous outflow under the influence of increasing progesterone, beta subunit of HCG levels, decreased episcleral venous pressure related to generalized reduction in peripheral vascular resistance. As the IOP is related to Blood Pressure, along with routine BP monitoring during pregnancy IOP should be measured to detect and diagnose pre-eclampsia and other ocular disorders at an early stage to reduce the risk of hypertension, retinopathy and loss of vision. We conclude that IOP measurement should be done along with routine ante-natal check-up as a part of it.

It is important to be aware of vision changes during pregnancy and to know what symptoms indicate a serious problem.

\section{Journal Papers:}

\section{References}

[1]. Pilas-Pomykalska M, Luczak M, Czajkowski J, Woznjak P and Oszwkowski. Changes in Intra Ocular Pressure during pregnancy, P Klin Oozna. 2004: 106(1-2 suppl): 238-9.

[2]. Giannina G, Belfort MA, Abadejos P, Dorman K. Comparison of IOP between normotensive and pre-eclamptic women in the peripartum period. Am J Obstet Gynecol 1997 May: 176 (5): 1052-5.

[3]. Sunness Js. The pregnant woman's eye. Surv.Ophthalmol 1998; 32: 219-38.

[4]. Ziai N, Org SJ, Khan AR, Brubaker RF. Beta- human Chorionic Gonadotrophin, progesterone and aqueous dynamics during pregnancy. Arch ophthalmol, 1994 Jun: 112 (6): 801-6.

[5]. Avasthi P,Luthra MC. Effects of sex hormones on intraocular pressure-Int Surg1967;48:350-5

[6]. Becker B. Friedenwald JS. Clinical aqueous outflow Arch ophthalmol 1953; 50: 557-71.

[7]. Imre J. Pregnancy and the eye, their endocrinological relations.XV Concilium Ophthalmol Egypt III. 1937; 213-26.

\section{Books:}

[8]. Moses RA, IOP. In: Moses RA.ED. Adler's physiology of the eye : clinical application $7^{\text {th }}$ ed. St. Louis Mosby 1981.

[9]. Carel RS, Korczyn AD, Rock M etal. Association between ocular pressure and certain health parameters. Ophthalmology 1984.

\section{Chapters in Books:}

[10]. Davis EA, Dana MR. Pregnancy and the eye, In; Albert DM, Jacobiek FA. Editors. Principles and practice of OphthalmologySecond Edition. Vol 5.W.B.Saunders Co. Philadelphia 2000; 4767-83.

[11]. Bill A. The role of ciliary body blood flow and ultra-filtration in aqueous humour formation, Exp Eye Res 1973; 16: 287-98.

[12]. Bill A. Blood circulation and fluid dynamics in the eye. Physiological Rev 1975; 55: 383-417. 